\title{
TTR
}

Traduction, terminologie, re?daction

\section{Valeur sémantique du verbe dans les collocations verbales spécialisées}

\section{Louise Larivière}

Volume 11, numéro 1, 1er semestre 1998

Diachronie et synchronie

Diachronics and Synchronics

URI : https://id.erudit.org/iderudit/037321ar

DOI : https://doi.org/10.7202/037321ar

Aller au sommaire du numéro

\section{Éditeur(s)}

Association canadienne de traductologie

ISSN

0835-8443 (imprimé)

1708-2188 (numérique)

Découvrir la revue

Citer cet article

Larivière, L. (1998). Valeur sémantique du verbe dans les collocations verbales spécialisées. TTR, 11(1), 173-197. https://doi.org/10.7202/037321ar

\section{Résumé de l'article}

Valeur sémantique du verbe dans les collocations verbales spécialisées - Le présent article se propose de spécifier la valeur sémantique du verbe qui fait partie des collocations verbales spécialisées (CVS) et de caractériser, de ce fait, la nature même de cette collocation afin d'en faciliter l'identification lors de la collecte des données et, par la suite, la consignation dans un répertoire. Pour ce faire, nous établissons d'abord la place qu'occupe la collocation ou syntagme semi-figé parmi les combinaisons lexicales; puis, nous précisons la nature de la collocation, tant en langue générale qu'en langue de spécialité, en faisant ressortir la diversité de ses acceptions et de ses appellations; et, enfin, nous décrivons les caractéristiques propres et communes des trois types de verbe qui entrent dans la composition des CVS : le verbe à valeur métaphorique, le verbe à sens plein et le verbe à sens vide. Le statut du verbe, ainsi établi, permet de déterminer la composition des collocations verbales, en particulier en français commercial.
Tous droits réservés @ TTR: traduction, terminologie, rédaction — Les auteurs, 1998
Ce document est protége par la loi sur le droit d'auteur. L'utilisation des services d'Érudit (y compris la reproduction) est assujettie à sa politique d'utilisation que vous pouvez consulter en ligne.

https://apropos.erudit.org/fr/usagers/politique-dutilisation/ 


\section{Valeur sémantique du verbe dans les collocations verbales spécialisées}

\section{Louise Larivière}

\section{Introduction}

Dans la conception d'un produit dictionnairique, en particulier d'un dictionnaire de collocations dont il sera question dans cet article, il importe de préciser le but poursuivi, qui est fonction du public visé, afin d'orienter la composition et le traitement des unités qui en font partie. Dans un précédent article (Larivière, 1998, p. 182-183), dans lequel nous décrivons la structure d'un thésaurus thématique et alphabétique des collocations verbales en français commercial (FC) que nous sommes en train de compiler, nous avons précisé ce but et ce public utilisateur : foumir un outil d'encodage fonctionnel, pratique et de consultation facile devant, à la fois, aider à la production rapide de textes commerciaux idiomatiques par des « langagistes n, des joumalistes, des gestionnaires ou autres, et à l'apprentissage de la langue française commerciale, qu'elle soit première ou seconde.

Pour atteindre ces objectifs, nous avons voulu inclure des combinaisons lexicales susceptibles de répondre aux besoins langagiers du public visé et fournir le plus de clés d'accès possible pour faciliter la consultation de ces combinaisons au sein du thésaurus. Aussi avons-nous proposé (p. 186-187) une macrostructure à trois volets : un volet 
conceptuel (liste thématique), un volet descriptif (listes alphabétiques) et un volet enumératif (index KWIC)'. Quant à la microstructure des collocations verbales (CV), nous l'avons décrite brièvement en donnant certaines caractéristiques du verbe et du nom qui entrent dans la composition de ces collocations. C'est pourquoi nous voulons, dans le présent article, préciser davantage la valeur sémantique du verbe qui fait partie des collocations verbales spécialisées (CVS) et, du même coup, caractériser la nature même de cette collocation afin d'en faciliter l'identification lors de la collecte des données et, par la suite, la consignation dans un répertoire.

Pour ce faire, nous allons d'abord situer la collocation ou syntagme semi-figé parmi les combinaisons lexicales, puis préciser la nature et la désignation de la collocation, tant en langue générale (LG) qu'en langue de spécialité (LS), en faisant ressortir la diversité de ses acceptions et de ses appellations et, enfin, décrire les trois valeurs sémantiques du verbe qui entre dans la composition des $\mathrm{CV}$ en $\mathrm{FC}$ et déterminer, par le fait même, la composition de la CVS.

\section{Place de la collocation parmi les combinaisons lexicales}

Les collocations sont des combinaisons de mots qui ont été longuement étudiées sans qu'il y ait eu unanimité sur la façon de les concevoir et de les dénommer. En considérant les types de groupements, que les divers

\footnotetext{
'La première partie LISTE THÉMATIQUE DES COLLOCATIONS VERBALES consiste en une classification de toutes les collocations verbales hiérarchisées en trois niveaux en fonction des caractéristiques que présentent les verbes de ces collocations : niveau sémantique où les verbes sont classés en fonction de certaines catégories préétablies, niveau stylistique où les verbes sont classés en fonction des aspects qu'ils présentent et niveau syntaxique où les verbes sont classés en fonction de leurs actants (sujet, verbe, circonstant) et sous-classés en fonction de leur constitution : verbes simples (unitermes et bitermes) et verbes complexes; 2) la deuxième partie LISTES ALPHABÉTIQUES DES COLLOCATIONS VERBALES comprend quatre listes dont deux avec entrées aux verbes (en français et en anglais) et deux avec entrées aux noms (en français et en anglais); 3) la troisieme partie INDEX KWIC DES COLLOCATIONS VERBALES consiste en la liste générale de toutes les collocations verbales (en français et en anglais) avec mise en vedette de chacun des termes pleins selon l'ordre alphabétique, d'où l'acronyme KWIC (key-word in context).
} 
ouvrages sur la question retiennent (v. par exemple Benson/Benson/lson (BBI), 1997 et Cohen, 1986), on s'aperçoit que la notion de collocation n'est pas partout la même. Aussi, avant de proposer une définition de la collocation, nous allons, d'abord, situer les collocations parmi l'ensemble des combinaisons lexicales.

Les combinaisons lexicales se retrouvent à divers niveaux de la chaîne parlée : au niveau du terme, au niveau du syntagme et au niveau de la phrase (v. Tableau 1 en annexe p. 193). À une première extrémité, au niveau du terme, se trouvent des combinaisons de mots qui correspondent à une notion. Ces combinaisons sont constituées soit de termes composés dont les éléments sont reliés par un trait d'unjon (ex. sous-louer), soit de termes complexes dont les éléments sont séparés par des blancs (ex. faire faillite). À l'autre extrémité, au niveau de la phrase, se trouvent des combinaisons de mots qui traduisent un énoncé complet. Ces combinaisons peuvent être soit figées à structure fixe (ex. Veuillez agréer mes salutations distinguées), soit libres à structure variable (ex. Le chèque n'a pas de provisions).

Entre les deux, au niveau du syntagme (au sens chomskyen de constituant de la phrase), on distingue généralement trois types de combinaisons en fonction de leur degré de figement (Hausmann, 1985, p. 1010) : syntagme figé ou locution, syntagme semi-figé ou collocation et syntagme [non-figé] ou association libre. De plus ces combinaisons sont caractérisées par des traits de cohésion et d'autonomie étroitement liés au trait de figement. En effet, dans un syntagme figé, le degré de cohésion entre les éléments est tel que le degré de figement est total puisqu'aucun élément ne peut s'insérer entre les éléments figés; de plus, le degré d'autonomie des éléments est nul puisque le sens individuel de chacun des éléments est perdu au profit du sens du tout au point qu'il n'y a pas de commutation possible des éléments (ex. donner un chèque en blanc à quelqu'un qui signifie le laisser libre de choisir, de décider). A l'opposé, dans le syntagme libre, la cohésion entre les éléments étant plus lâche, d'autres éléments peuvent se joindre au syntagme; de plus, leur autonomie sémantique est respectée de sorte que le sens du tout égale la somme du sens de chacun d'eux - on parle, dans ce cas, du caractère 
compositif ${ }^{2}$ des éléments - (ex. dans déchirer un chèque, le sens du verbe déchirer ajouté à celui du nom un chèque donne le sens global de déchirer un chèque); finalement, chacun des éléments a la possibilité de se combiner avec plusieurs autres éléments.

Le troisième type de syntagme, le syntagme semi-figé ou collocation, qui nous intéresse plus particulièrement, possède des caractéristiques propres aux deux autres syntagmes et constitue, de ce fait, un syntagme hybride. D'une part, la collocation acquiert un certain degré de figement une fois qu'elle est, bien qu'arbitrairement, consacrée par l'usage. Ainsi, elle constitue un phènomène de langue et non un phénomène de parole qui doit s'apprendre comme tout autre phénomène de langue (Hausmann, 1985, p. 1010). De ce point de vue, elle se rapproche du syntagme figé. D'autre part, elle est dite semi-compositive ${ }^{2}$ en ce sens que ses termes composants n'égalent que partiellement le tout. De ce point de vue, elle se rapproche du syntagme libre bien que son degré de combinabilité soit plus restreint. Une autre caractéristique qui la rapproche du syntagme libre est le fait de pouvoir se présenter sous diverses structures syntaxiques : ex. encaisser un chèque (verbe à l'infinitif = forme canonique), j'encaisse un chèque (verbe conjugué à la voix active), le chèque a été encaissé (verbe conjugué à la voix passive), le chèque encaissé (nom + participe adjectif), en encaissant son chèque (participe présent), le chèque que j'ai encaissé (antécédent + relative), l'encaissement du chèque (déverbal).

La collocation présente donc des caractéristiques mitigées : elle est une combinaison plus ou moins figée par l'usage, son degré de cohésion est plus ou moins lâche, son degré de combinabilité est restreint et ses termes cooccurrents ont également une autonomie sémantique limitée vu qu'ils n'acquièrent un sens déterminé que par leur combinaison avec le terme noyau qui, lui, garde son autonomie. Ainsi dans l'exemple toucher un chèque, le verbe toucher a un sens figuré et signifie encaisser de par sa combinaison avec le nom chèque, alors que ce dernier garde son sens propre. Toutefois le degré d'affinité entre les éléments d'une collocation est tel que l'on peut dire de ces derniers qu'ils sont $\alpha$ des

${ }^{2}$ D'autres parleront de compositionnalité et du caractère compositionnel ou semicompositionnel des éléments. 
mots qui vont si bien ensemble $*^{3}$ au point de $\alpha$ donne[r] l'impression du "déjà vu" "(Bally, 1951, p. 70). Nous pouvons maintenant définir la collocation comme suit :

(1) Combinaison semi-figée, récurrente et arbitraire, d'unités lexicales qui ont développé une affinité ou relation privilégiée consacrée par l'usage, qui ont une combinabilité restreinte et un statut distinct, l'une étant sémantiquement autonome et l'autre n'acquérant son sens qu'au sein de la combinaison, et dont la somme n'égale que partiellement le sens individuel de chacune.

L'identification des combinaisons lexicales n'est, toutefois, pas toujours aussi simple. Il arrive, parfois, que l'on hésite à classer certaines combinaisons dans tel ou tel groupe. Ainsi, la combinaison déposer son bilan peut, à première vue, sembler être une collocation. Toutefois, cette combinaison peut globalement être substituée à une combinaison synonyme se déclarer en faillite. Elle n'est donc pas une collocation mais un syntagme figé ou locution dont le sens global n'égale pas le sens individuel des parties. De même, est-ce que la combinaison prendre une décision est une collocation ou un terme complexe? En fait, elle se rapproche plus du terme complexe puisqu'elle peut être substituée à un uniterme décider. Toutefois, il n'est pas toujours évident de statuer sur les combinaisons formées d'un verbe vide (ex. prendre, faire, etc.) et d'un nom complément. En effet, est-ce que les combinaisons faire l'achat d'une maison et faire cession de ses biens sont toujours substituables à acheter une maison et céder ses biens? C'est à voir. Par ailleurs, est-ce que les combinaisons vendre des actions et payer une facture, où les verbes vendre et payer gardent leur sens plein, pourraient être considérées comme des collocations, en supposant que cette dernière soit définie autrement ou qu'elle englobe d'autres considérations? Avant de répondre à cette question, il y a lieu d'examiner les distinctions faites à propos des collocations dans divers écrits.

${ }^{3}$ Cette phrase a été empruntée au professeur Brian Rainey de l'Université de Regina (SK), qui l'a luí-même empruntée à une chanson des Beatles, Michelle, et qu'il utilise dans ses cours pour faire comprendre ce qu'est une collocation. 


\section{Diversité des acceptions et des désignations des collocations}

Dans les divers écrits traitant des collocations, on s'entend généralement sur le caractère arbitraire et récurrent des collocations, sur les liens d'affinité ou privilégiés que les composants entretiennent entre eux de même que sur le statut inégal de ces demiers. Ce sur quoi on s'entend le moins, c'est sur la composition des collocations, sur leur traitement à l'intérieur des répertoires linguistiques de même que sur leur désignation. Tout comme les untess lexicales appartiennent soit à la LG soit à la LS, de même les combinaisons lexicales appartiendront à l'une ou à l'autre langue pour former deux groupes distincts : les collocations en langue générale (CLG) et les collocations en langue de spécialité (CLS). Toutefois, il importe de préciser que les CLG, tout comme les unités lexicales consignées dans les dictionnaires de langue générale, comprennent à la fois des mots de la LG et de la LS, alors les CLS n'appartiennent qu'à un domaine de spécialité.

\subsection{Collocations en langue générale (CLG)}

En LG, la distinction la plus connue est celle qui est faite entre la collocation lexicale (CL) et la collocation grammaticale (CG). Pour Hausmann (1985, p. 1010), la collocation (qui n'est pas autrement précisée) est une " combinaison caractéristique de deux mots dans une des structures suivantes :
a) substantif + adjectif (épithète)
b) substantif + verbe
c) verbe + substantif (objet)
d) verbe + adverbe
e) adjectif + adverbe
f) substantif + (prép.) + substantif .

Ces deux mots n'ont pas un statut égal : l'un est autonome au plan sémantique (la base), l'autre ajoute une caractérisation au premier sans en modifier l'identité (le collocatif) et a ne reçoit son identité sémantique que par la collocation *. Comme pour Hausmann (p. 1011), la collocation * est avant tout un phénomène de production de textes (et non de compréhension) ", l'entrée dans les dictionnaires de collocations se fera 
à la base puisque cette dernière est connue et que c'est le collocatif que l'on recherche.

Benson/Benson/Ilson (BBI, 1997, p. xv) reconnaissent deux grandes catégories de collocations : les collocations grammaticales et les collocations lexicales. Pour eux, une collocation grammaticale est " a phrase consisting of a dominant word (noun, adjective, verb) and a preposition or grammatical structure such as an infinitive or clause $*(p$. xv). Ils reconnaissent, par ailleurs, sept structures de collocations lexicales ( $\mathrm{p} . \mathrm{xxx}$ ) qui s'identifient à celles de Hausmann à l'exception de la structure verbe + substantif (objet) qui se divise en deux sous-structures en fonction du sens du verbe : l'une où le verbe dénote une création (creation) ou une activité (activation) et l'autre où le verbe dénote une suppression (eradication) ou une invalidation (nullification). Dans leur dictionnaire, les entrées sont, avant tout, constituées de noms, d'adjectifs et de verbes, quelques-unes d'adverbes et de prépositions et se présentent comme suit : 1) les types de collocations sont indiqués par des exemples plutôt que par des énoncés; 2) les collocations lexicales précèdent les collocations grammaticales; la variété d'anglais est indiquée par $\mathrm{AE}$ (anglais américain) et par BE (anglais britannique); 3) les locutions idiomatiques ne sont pas consignées à l'exception de celles qui se situent à la limite des collocations et des locutions (transitional phrases); 4) un certain nombre de groupes figés qui ne font pas partie des collocations lexicales ni grammaticales décrites sont également consignés sous la rubrique divers.

Dans le Dictionnaire explicatif et combinatoire (DEC) de Mel'cuk et al. (1984), on ne parle pas de CLG, de CLS, de CL, de CG ni même de collocation. On parle plutôt de cooccurrence lexicale qui consiste en * la capacité des lexèmes de se combiner en syntagmes pour exprimer un sens donné " et plus particulièrement de cooccurrence lexicale restreinte qui se réalise " si un lexème $A$ signifiant ' $A$ ' et un lexème B signifiant ' $B$ ' ne peuvent se combiner pour exprimer le sens composé ' $A+B$ ' " (p. 4). On traite le lexème vedette (ou mot-clé), ainsi que chaque locution figée ou semi-figée à laquelle ce lexeme peut participer, sous deux aspects : on en explicite le sens et on en détaille toutes les combinaisons possibles (d'où le titre de l'ouvrage). Ce traitement se fait a à tous les niveaux linguistiques, en particulier, la cooccurrence lexicale, énumérant ainsi toutes les locutions non libres qui 
contiennent le lexème vedette, ce qui équivaut à décrire tous les cas de cooccurrence lexicale restreinte (p. 4). Pour tout article de dictionnaire, le DEC présente la structure suivante (p. 5) : 1) une définition du lexème, 2) un schéma de régime qui représente tous les actants syntaxiques du régime et 3) des fonctions lexicales (FL) (p. 6). La fonction lexicale s'écrit comme suit : $f(X)=Y$ \& où $f$ est la $F L, X$ est son argument (un lexème ou bien une locution), et $Y$ est la valeur de la FL $f$ pour cet argument, c'est-à-dire l'ensemble des expressions linguistiques qui peuvent exprimer le sens ou le rôle syntaxique donné (noté par $\mathrm{f}$ ) auprès de $\mathrm{X} *$ (p. 6) comme dans l'exemple suivant : soit la formule : $\boldsymbol{R e a l}_{2}$ (ordre) $=$ exécuter où Real $_{2}$ est la fonction qui représente un verbe ayant le sens de 'réaliser' exerçant son action sur le mot-clé ordre et prenant la valeur de exécuter pour donner l'expression exécuter un ordre. Dans Mel'cuk/Clas/Polguère (1995), cependant, on introduit le terme phrasème (locution prise dans une acception bien spécifique, p. 16) et l'on définit le semi-phrasème (= collocation) comme « un phrasème $\mathrm{AB}$ au signifié 'AC' ou 'BC' qui inclut le signifié de l'un des constituants, alors que l'autre soit ne garde pas son sens, soit - même s'il garde son sens - n'est pas sélectionné librement $n$ (p. 46).

\subsection{Collocations en langue en spécialtié (CLS)}

Parmi les répertoires qui traitent des collocations en langue de spécialité, citons les ouvrages de Cohen $(1983,1986)$ et de Lainé (1993). Cohen, toutefois, parle de cooccurrence et non de collocation et sa démarche suit celle de Mel'cuk et al. (1984), surtout dans sa thèse de maîtrise (1983). Dans la version publiée de son étude (1986), l'approche est plus conviviale : dans ume grille de coordonnées, les cooccurrents sont classés par catégories grammaticales (substantifs, verbes, etc.) et présentés en abscisse, alors que les différents stades du cycle économique sont présentés en ordonnée. Tant chez Cohen que chez Lainé, la vedette est au nom suivie d'une définition. Il en est de même chez Pesant/Thibault (1993) qui, par ailleurs, préferent aussi utiliser le terme de cooccurrence pour la langue de spécialité : « [...] nous préférons employer, en langue de spécialité, le terme de cooccurrence et son dérivé cooccurrent à ceux de collocation et de collocatif et collocateur. Tout comme on distingue la terminologie de la lexicographie, nous avons pensé distinguer la cooccurrence de la collocation "(p. 25). La distinction entre collocation (LG) et cooccurrence (LS) est intéressante, bien que cooccurrence nous 
semblerait plus approprié comme terme générique pour regrouper tous les types de combinaisons lexicales.

Dans d'autres études, on utilise le terme de phraséologismes (Blais, 1993; Pavel, 1993) pour désigner les collocations en langue de spécialité. Pour Blais (p. 52) un phraséologisme est une " [c]ombinaison d'éléments linguistiques propre à un domaine de spécialité, dont l'un est un terme noyau, qui sont liés sémantiquement et syntaxiquement et pour lesquels il existe une contrainte paradigmatique [...] faisant en sorte qu'il y ait un nombre limité de substitution des éléments de la combinaison sans qu'il y ait un changement des idées véhiculées ". Toutefois, le terme phraséologisme nous semble trop associé aux locutions ou syntagmes figés pour être utilisé pour désigner les combinaisons lexicales en LS.

On utilise, par ailleurs, l'expression combinaison lexicale spécialisée (L'Homme, 1997; Meynard, 1998; L'Homme/Meynard et Bertrand (v. le présent numéro). L'Homme (p. 15) souligne certains problèmes que posent les combinaisons lexicales : manque d'uniformité dans leur désignation, leur recensement et leur codification. De plus, les modèles terminologiques généralement proposés sont fondés sur le concept et sont articulés autour du nom, ce qui rend difficile une description linguistique qui se définit par rapport à un usage selon un modèle défini : création d'une base de données parallèle décrivant les acceptions techniques des unités verbales et établissement d'un lien entre cette base verbale et une base de données terminologiques classique ( $p$. 18). Aussi réserve-t-elle le terme de collocation pour les syntagmes semifigés appartenant à la LG et la désignation de combinaison lexicale spéciallsée $(C L S)^{4}$ pour ceux qui relèvent d'une LS. Ainsi, il y aurait un vocabulaire distinct pour les combinaisons lexicales en LG et en LS, comme il y en a un pour les unités lexicales (le mot en LG et le terme en LS). Toutefois, l'appellation combinaison lexicale spécialisée nous apparaît trop générale pour servir de désignation à la notion que l'on veut exprimer, soit une combinaison particullère de termes spécifique d la $\boldsymbol{l} \boldsymbol{S}$, et peut entraîner, de ce fait, de la confusion. Il reste donc à trouver un

${ }^{4}$ Il arrive que notre sigle CLS (collocation en langue de spécialité) soit homonyme avec le sigle CLS (combinaison lexicale spécialisée) de Marie-Claude L'Homme. Question de pur hasard. 
terme approprié pour désigner la combinaison lexicale en langue de spécialité, s'il y a vraiment lieu de le faire.

En résumé, les combinaisons lexicales du type syntagmes semifigés sont appelées de diverses façons dans les études que nous avons examinées : collocations, cooccurrences ou semi-phrasèmes en LG et cooccurrences, phraséologismes ou combinaisons lexicales spécialisées en LS. Cette liste n'est, toutefois, pas exhaustive puisque d'autres appellations ont aussi été proposées. Pour ce qui est des collocations en LG, on distingue entre les collocations lexicales et les collocations grammaticales.

Quant à nous, nous conservons le terme de collocation pour les combinaisons lexicales tant en LG qu'en LS en lui ajoutant la caractérisation appropriée : collocation en langue générale (CLG) et collocation en langue de specialite (CLS). Nous adoptons cette position pour ne pas à avoir à multiplier indament les termes, même si les collocations verbales en FC, objet de nos recherches, ont une acception plus large que celle de la collocation définie plus haut et que le traitement que nous en faisons diffère des traitements plus classiques. Par ailleurs, nous maintenons la distinction entre les CL et les CG pour les CLG (v. les exemples prendre une décision et décider de au Tableau 1). Pour ce qui est des CLS, nous les subdivisons en fonction du terme noyau : verbe, nom, adjectif, adverbe (v. Tableau 1). Pour le moment, nous ne nous sommes attardée qu'aux collocations verbales où le verbe est le terme noyau autour duquel gravitent ses actants sujet, objet, circonstant (Larivière, 1998, p. 181).

\section{Valeur sémantique du verbe dans les collocations verbales}

Les collocations spécialisées ayant le verbe comme terme noyau ont été celles qui ont le plus fait l'objet d'études récentes (Bodson, 1998; Larivière, 1998; L'Homme, 1993, 1995, 1997), et ce, pour les mêmes raisons : problèmes que posent les verbes polysémiques, rareté des études et des répertoires sur le sujet et surtout nécessité de fournir des outils aux " langagistes " qui ont à effectuer des travaux d'encodage (traduction, rédaction) où l'utilisation du verbe approprié, accompagnant un cooccurrent (nom sujet, nom objet ou circonstant), témoigne d'une 
parfaite maîtrise des collocations ainsi que d'une connaissance idiomatique de la langue et d'un niveau de langue relevé :

$$
\begin{aligned}
& \text { SN + SV : des capitaux fuir } \\
& \text { SV + SN : dresser un budget } \\
& \text { SV + SP : s'ouvrir à la compétition } \\
& \text { SV + SA : se constituer en société }
\end{aligned}
$$

Par ailleurs, l'écueil majeur rencontré lors de la collecte des données en vue de la constitution de notre corpus en $\mathrm{FC}$, fut de déterminer quelles combinaisons constituaient des collocations verbales (CV) et lesquelles n'en constituaient pas. Nous avons déjà traité de la forme que devait prendre le verbe : un uniterme (ex. annuler), un biterme (souslouer) ou un terme complexe (faire faillite) au mode infinitif; quant au nom qui accompagne le verbe, il devait être un terme du domaine d'étude (Larivière, 1998, p. 192-193). Il importait, cependant, de déterminer le statut du verbe (ou sa valeur sémantique), au sein des $\mathrm{CV}$, en fonction de critères bien définis, afin de faciliter l'jdentification des collocations.

Deux auteurs ont déjà établi des critères pour déterminer si un syntagme, formé d'un verbe et d'un nom objet, constitue ou non une collocation. Le premier, Howarth (1993, p. 4, cité par Fontenelle, 1994, p. 48), distingue trois types de collocations (restricted collocations ${ }^{5}$ ) dont la structure syntagmatique se compose d'un verbe et d'un complément d'objet direct, qui sont des combinaisons semi-figées et dont la combinabilité est restreinte : les delexical collocations qui se composent de verbes à sémantisme vide (tels have, make, do, get), les collocations avec un figurative verb (adopt a policy) et les collocations techniques ou spéclalisées (snuff off a candle).

Le second, Benson (1989, p. 4-5) examine les trois cas suivants où le verbe est également suivi d'un objet direct. Dans le premier cas (p. 4), il s'agit de verbes qui peuvent être suivis d'une quantité quasi illimitée de compléments et qui doivent, par conséquent, être éliminés des collocations qui sont à combinabilité restreinte : buy, carry, cause,

${ }^{5}$ En fait ces restricted collocations sont des collocations au sens propre du terme qui s'opposent aux free collocations qui sont des combinaisons libres. 
create, destroy, hate, hit, kick, like, make, see, sell, take, turn, etc. Toutefois, ces verbes peuvent faire partie de syntagmes figés (ex. make a mountain out of a molehill) ou se trouver a la limite entre les syntagmes figés et les collocations (ex. take one's time $=$ to act slowly). Aussi đésigne-il ces dernières combinaisons comme étant des transitional collocations (p. 5). Par contre, lorsque ces verbes sont rattachés à des noms appartenant à une LS, ces verbes peuvent former avec ces noms des collocations (ex. create a file). C'est ce qu'il appelle des technical collocations (p. 5) qui devraient figurer dans un dictionnaire de collocations.

Benson mentionne une autre catégorie de verbes (p. 5) qui peuvent être suivis d'un grand nombre de compléments, néanmoins limités sémantiquement (ex. run, au sens de direct ou manage et delete). Les combinaisons formées avec ces verbes ne doivent pas théoriquement faire partie d'un dictionnaire de collocations sauf si elles constituent des technical collocations (syntagmes formés d'un verbe et d'un nom d'une LS) ou si elles sont composées de noms polysémiques qui sont combinés à d'autres verbes avec lesquels ils forment déjà des collocations :

$$
\begin{aligned}
& \text { run a business (firm) } \\
& \text { conduct/do/transact business (commerce) }
\end{aligned}
$$

Dans cet exemple le terme business est polysémique. Comme ce terme (au sens de commerce) forme déjà des collocations avec les verbes conduct, do et transact, ce même terme (au sens de firm) formera une collocation avec le verbe run. Finalement, Benson mentionne une troisième catégorie de verbes (p. 5) qui se combinent avec un petit groupe de noms, bien souvent dans un vocabulaire technique (ex. debug a program). Ces combinaisons doivent évidemment faire partie d'un dictionnaire de collocations.

Quant à nous, nous avons identifié trois types de verbes pouvant se combiner avec tous leurs actants : les verbes à valeur metaphorique, les verbes à sémantisme plein et les verbes à sémontisme vide ( $\mathrm{v}$. Tableau 1 où, pour des raisons d'économie d'espace, nous les avons nommés verbe figure : toucher un chèque, verbe plein : encaisser un chèque et verbe vide : faire un chèque). Nous exposerons donc les caractéristiques 
de chacun de ces trois types de verbes et, par le fait même, nous préciserons la nature de la collocation verbale spécialisée (CVS).

\subsection{Le verbe à valeur métaphorlque}

Le verbe à valeur métaphorique est un verbe qui n'a pas d'identité sémantique autonome et dont le sens ne se précise que par l'actant avec lequel il se combine comme dans les exemples qui suivent attestés dans le GRE (1989) ou le NPR (1995) comme ayant un sens figuré (F) ou abstrait (A) :

(5) absorber des économies $F=$ faire disparaître en utilisant

$\begin{array}{ll}\text { casser les prix } & \begin{array}{c}\mathrm{A}=\text { provoquer une brusque chute des } \\ \text { prix sur le marché }\end{array} \\ \text { clore un marché } & \mathrm{F}=\text { achever, arrêter, finir, terminer } \\ \text { engloutir des sommes } & \mathrm{F}=\text { dépenser rapidement } \\ \text { éponger une dette } & \mathrm{F}=\text { résorber } \\ \text { éteindre une dette } & \mathrm{F}=\text { faire cesser d'exister } \\ \text { lever des impôts } & \mathrm{F}=\text { percevoir, recueillir }\end{array}$

Il est en de même pour d'autres verbes qui ne sont pas identifiés comme étant au figuré dans le GRE mais qui se comportent de la même façon que s'ils l'étaient.

$$
\begin{aligned}
& \text { arrêter un compte } \\
& \text { comprimer des dépenses } \\
& \text { épuiser un stock } \\
& \text { lever des titres } \\
& \text { verser un dividende }
\end{aligned}
$$

En réalité, ces verbes polysémiques prennent un sens particulier du fait de leur combinaison avec un terme appartenant à une langue de spécialité. Comparons le même verbe avec un mot de LG et un terme de LS :

$$
\text { verser du lait et verser un dividende }
$$

où verser $a$, dans le premier exemple, le sens de faire couler un liquide dans un récipient qu'on incline et, dans le deuxième, le sens de payer. Par conséquent, la combinaison verser du lait est une association libre 
parce que le sens du verbe verser est tel qu'il peut s'associer une grande quantité de compléments. Par contre, verser un dividende est une collocation parce que l'actant du verbe, le nom dividende, est un terme de LS qui confère à ce verbe un sens particulier et parce que verser au sens de payer a une capacité restreinte de commutation.

Il ne faut pas s'étonner de retrouver de telles métaphores, qui sont nombreuses, en français commercial. Philippe Dominique (1971, p. 55), cité par Cohen (1986, p. iii), constate que :

La où le profane ne songerait qu'à des termes antonymiques tels que hausse/baisse, gain/perte, ces deux vocabulaires du commentaire boursier [le français et l'anglais] multiplient les métaphores, varient les constructions syntaxiques, surprennent par des collocations syntagmatiques inusitées, et constituent une véritable stylistique.

Pour sa part, Cohen (1986, p. iii) parle d'un * foisonnement de métaphores et d'images toujours plus étonnantes "dans le domaine du commentaire économique, boursier ou de conjoncture, et donne plusieurs exemples. Elle relève, entre autres, sous action (Cohen, 1983, p. 43) et sous prix (p. 185-187) :

UNE ACTION + V

gagner du terrain, se réveiller

chuter, s'effriter, faiblir, s'effondrer stagner, végéter

(9) UN PRIX + V

grimper, s'alourdir, bondir, s'envoler, s'emballer, flamber, monter en flèche, décoller, s'enfler, se gonfler, exploser chuter, s'effondrer se détendre

V + UN PRIX gonfler, enfler, attiser

Cohen (1986, p. iii) ne considère pas, toutefois, ces combinaisons comme étant des collocations techniques. Elle les envisage, plutôt, comme $\alpha$ des syntagmes tirés de la langue générale et qui, greffés aux termes spécialisés, prennent un sens nouveau $"$. Ces métaphores au sein des collocations peuvent, cependant, aux dires de Meyer/Mackintosh (1996, 
p. 5) jouer un rôle important en terminologie en foumissant de l'information sur l'ensemble de la structure conceptuelle d'un domaine de même que sur les concepts eux-mêmes.

Donc, peut être considérée comme un premier cas de CVS la combinaison lexicale formée d'un verbe à valeur métaphorique et d'un terme spécialisé (actant du verbe) qui lui confère son identité sémantique.

\subsection{Le verbe à sémantisme plein}

Parallèlement aux verbes à sens figuré, il existe des verbes à sémantisme plein, soit des verbes qui constituent des termes renvoyant à une notion sans avoir besoin du nom pour lui donner une identité sémantique. De plus, ces verbes sont reconnaissables au fait qu'ils peuvent se paraphraser par une expression qui contient leur déverbal.

$$
\begin{aligned}
& \text { barrer un chèque }=\text { marquer d'une barre } \\
& \text { déposer de l'argent (a la banque) }=\text { mettre en dépôt } \\
& \text { liquider des biens = soumettre à une liquidation } \\
& \text { retirer de l'argent (à la banque) }=\text { effectuer un retrait } \\
& \text { soustraire ses frais généraux }=\text { retrancher par soustraction }
\end{aligned}
$$

Même des verbes très courants, comme payer et vendre, qui ont un sémantisme plein et qui ont toujours un même signifié, quel que soit l'actant avec lequel ils se combinent (donc étant monosémiques), peuvent former avec ces derniers, s'ils sont des termes d'une LS, des CVS. Alors que ces verbes peuvent, en LG, se combiner avec une grande quantité de termes, ils ont, en français commercial une combinabilité plus restreinte, ce qui est une caractéristique d'une collocation.

$$
\begin{aligned}
& \text { payer des dommages-intérêts } \\
& \text { payer une facture } \\
& \text { payer des impôts } \\
& \text { payer une indemnité }
\end{aligned}
$$

vendre des actions vendre des obligations vendre des titres

${ }^{6} \mathrm{Ce}$ qui signifie : revêtir le recto d'un chèque de deux barres parallęles dans le but de subordonner le paiement du chèque à l'intervention d'une banque (GRE, 1989, $s v$ chèque et barrer). 
payer un intérêt

payer une rente

payer une taxe vendre au comptant

vendre à crédit

vendre au détail

vendre en gros

vendre à perte

vendre à prix coûtant

vendre au prix fort

vendre au rabais

vendre à réméré

vendre en solde

vendre à terme

Il existe une autre caractéristique de ces verbes : ils doivent exprimer une activité propre à la LS. C'est pourquoi la combinaison déchirer un chèque ne peut être une collocation. D'abord, le verbe déchirer n'est pas un verbe à sens plein tel que défini plus haut et il n'exprime pas une activité propre au FC.

Donc - et ceci répond à la question posée au point 2 - peut être considérée, comme un deuxième cas de CVS, la combinaison lexicale formée d'un verbe à sémantisme plein et d'un terme spécialisé (actant du verbe), Je verbe étant sémantiquement autonome et monosémique, ayant une combinabilité restreinte et exprimant une activité propre à la LS ${ }^{7}$.

Il arrive, toutefois, que certains verbes soient homonymiques au sein d'une même LS et qu'ils aient, quelquefois mais rarement, tantôt un sens figuré, tantôt un sens plein comme dans les exemples suivants :

SENS FIGURÉ

engager des dépenses
SENS PLEIN

engager du personnel

${ }^{7}$ Clas $(1984$, p. 580) exclut, quant à lui, les verbes à sémantisme plein comme élément d'une collocation. Ainsi, pour lui, aciduler une solution, alcooliser une solution et alcoyler un métal sont des " cooccurrences libres $n$. Pour nous, ces combinaisons seraient des collocations parce que les verbes aciduler, alcooliser et alcoyler (ou alkyler) remplissent toutes les caractéristiques que nous avons établies pour la deuxième catégorie de verbes (ceux à sémantisme plein) devant constituer avec leurs actants des collocations verbales spécialisées. 
enregistrer un déficit

exporter une innovation enregistrer une société

exporter des produits bruts

La nature homonymique de ces verbes va, en quelque sorte, à l'encontre des critères que nous avons établis pour caractériser le verbe à sens plein qui, comme nous l'avons dit plus haut, a toujours le même signifié quel que soit l'actant avec lequel il se combine. Ceci est vrai pour un verbe comme vendre qui signifie toujours en FC : passer un contrat entre une personne $\mathrm{X}$ qui transfere un bien $\mathrm{Y}$ à une autre personne $\mathrm{Z}$ moyennant un prix en argent $W$ que $Z$ s'oblige à payer $\mathbf{a} X^{8}$. Mais ce n'est pas le cas pour tous les verbes comme en témoignent les verbes homonymiques. Aussi faudra-t-il considérer les verbes à sémantisme plein qui ont une variante homonymique à sens figuré comme des verbes appartenant à un sous-groupe de cette catégorie de verbes. Ainsi, par exemple, le verbe enregistrer dans la collocation enregistrer une société appartiendra à un sous-groupe de verbes à sens plein.

\subsection{Le verbe à sémantisme vide}

Les verbes à sémantisme vide sont des verbes dont le signifié s'identifie à la classe sémantique à laquelle ils appartiennent comme la possession (avoir, donner, posséder, prendre, rendre) et la réalisation (effectuer, entreprendre, établir, faire, mettre).

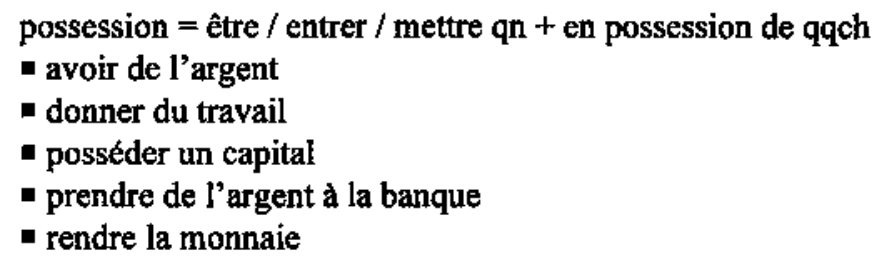

(14) réalisation

- effectuer un achat

- entreprendre une étude de marché

- établir un accord

\footnotetext{
- Cette définition s'inspire de celle du terme vente qui apparaît a l'article 1708 du Code civil du Québec.
} 
- établir des relations d'affaires

- établir une facture

- faire des achats

- faire un gain

- mettre une annonce

- mettre une somme sur un compte

De plus, les verbes à sémantisme vide, tout comme les verbes à sémantisme plein, se doivent d'être accompagnés d'un actant appartenant à une LS, exprimer une activité propre à la LS et avoir une combinabilité restreinte.

Donc peut être considérée, comme un troisième cas de CVS, la combinaison lexicale formée d'un verbe à sémantisme vide et d'un terme spécialisé (actant du verbe), le verbe s'identifiant à une classe sémantique, exprimant une activité propre à la LS et ayant une combinabilité restreinte.

\section{Conclusion}

Cette étude nous a d'abord permis de situer la collocation parmi l'ensemble des combinaisons lexicales, soit au niveau du syntagme, entre le terme (composé et complexe) et la phrase (figée et libre), et de la distinguer des autres syntagmes, figés et libres. Elle a également soulevé la question de la diversité des acceptions et des appellations entourant ce syntagme semi-figé qu'est la collocation pour en arriver à nous interroger sur la nécessité d'avoir un terme spécifique pour le désigner en langue générale et un terme spécifique pour le désigner en langue de spécialité. D'ici à ce qu'il y ait unanimité sur la question, nous avons préféré, pour ne pas multiplier les termes et entraîner de la confusion, caractériser tout simplement le terme collocation. Ainsi, nous proposons d'utiliser les appellations collocation en langue générale (CLG) et collocation en langue de spéclalté (CLS) en ayant soin, en les utilisant, de préciser la nature et le traitement de chacune d'elles.

Nous avons ensuite précisé la valeur sémantique du verbe qui fait partie des collocations verbales spéciallsées (CVS). Trois types de verbes, ayant des caractéristiques propres et communes, ont été présentés : le verbe à valeur métaphorique, le verbe à sémantisme plein tet le verbe 
à sémantisme vide. En établissant ainsi le statut du verbe, nous avons pu, du même coup, déterminer la composition de la CVS, ce qui en facilitera l'identification à l'étape de la collecte des données et la codification à celle de la consignation dans un répertoire.

Toutefois, compte tenu des considérations que nous avons faites à propos des CVS, nous ne pouvons maintenir, dans son entièreté, le libellé de la définition que nous avons utilisé plus haut car il est trop restreint pour englober toutes les caractéristiques des CVS. En effet, le trait de statut inégal des éléments de la collocation de même que celui de leur non-compositivité ne s'appliquent qu'à l'une des formes de CVS, soit la première. Aussi, nous devons restreindre la définition de la collocation, terme hyperonyme ou superordonné, de façon à la rendre suffisamment générale pour inclure les termes hyponymes ou subordonnés qui apparaissent dans la structure hiérarchique proposée au Tableau 1. Aussi, nous redéfinisssons la collocation comme suit :

$$
\begin{aligned}
& \text { collocation } \\
& \text { Combinaison semi-figée, récurrente et arbitraire, d'unités } \\
& \text { lexicales qui ont développé une affinité ou relation privilégiée } \\
& \text { consacrée par l'usage et dont la capacité combinatoire est } \\
& \text { limitée. }
\end{aligned}
$$

Par la suite, le terme de CLS, hyponyme du terme collocation, se définira comme suit :

\section{collocation en langue de spécialité (CLS) \\ Collocation formée d'un terme noyau, consistant en un syntagme verbal, nominal, adjectival ou adverbial, et de termes cooccurrents qui appartiennent à une langue de spécialité.}

Quant à la CVS, hyponyme de la CLS, nous en proposons la définition suivante :

\section{collocation verbale spécialisée (CVS)}

Collocation spécialisée formée d'un verbe et de l'un de ses actants, le verbe ayant soit un sens figuré sans identité autonome, soit un sens plein à caractère généralement monosémique, soit un sens vide rattaché à une classe sémantique, tout en reflétant, 
dans les deux derniers cas, une activité propre à la langue de spécialité.

Comme le trait * termes cooccurrents qui appartiennent à une langue de spécialité » fait partie de la définition de la CLS, nous n'avons pas à répéter ce trait dans la definition de la CVS qui est en relation d'inclusion avec la CLS. De même, nous n'avons pas à répéter le trait de * capacité combinatoire limitée » qui apparait dans la définition de la collocation, ni dans la définition de la CLS, ni dans celle de la CVS, également à cause de la relation d'inclusion qui existe entre la CLS, la CVS et la collocation. Aussi, il apparaît manifeste qu'un système de notions qui fait appel à une structure hiérarchique laquelle s'appuie sur une relation d'inclusion, facilite la définition des termes et évite la répétition des traits redondants.

Université Concordia 
Tableau 1 : Combinaisons lexicales

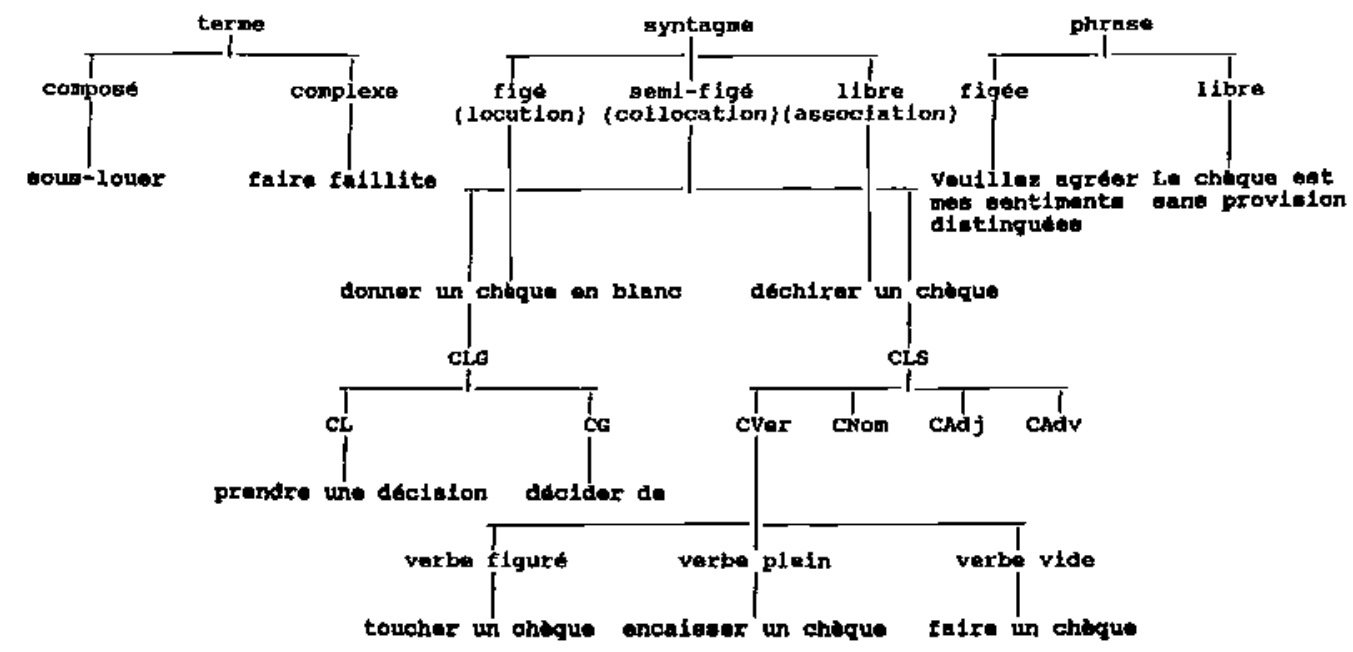

CLG = collocations en langue genarale

CL - collocationg lexicales

$C 6$ - collocations grampaticales cvor - collocationd verbalea

cNom = collocatlons nowinales

CAdf = collocations adjectivales

cAdv - collocations adverblales 


\section{Références}

BALLY, Charles (1951). Traité de stylistique française. $3^{\mathrm{e}}$ édition. Paris, Klincksieck / Genève, Georg \& Cie. 2 vol.

BBI (1997) = BENSON, Morton, BENSON, Evelyn et Robert ILSON (1997). The BBI Dictionary of English Word Combinations. Revised Edition. Amsterdam / Philadelphia, John Benjamins Publishing Company.

BENSON, Morton (1989). " The structure of the collocational dictionary ». International Journal of Lexicography, 2, 1, pp. 1-14.

BLAIS, Esther (1993). " Le phraséologisme. Une hypothèse de travail ". Terminologies nouvelles, 10, pp. 50-56.

BODSON, Claudine (1998). « Comparaison de deux méthodes de description des verbes en vue d'applications en traduction automatique : les verbes de langue générale et les verbes spécialisés \%. Traduction et langues de spécialité : approches théoriques et considérations pédagogiques, sous la direction de Zélie Guével et Egan Valentine. Québec, CIRAL. (Publication B; 214). pp. 139-154.

CLAS, André (1984). * Collocations et langues de spécialité ". Meta, 39, 4, pp. 576-580.

COHEN, Betty (1983). Lexique de cooccurrents. Bourse et conjoncture économique. Mémoire présenté à la Faculté des études supérieures en vue de l'obtention du grade de Maître ès Arts. Université de Montréal, décembre 1983.

COHEN, Betty (1986). Lexique de cooccurrents. Bourse et conjoncture économique. Montréal, Linguatech.

DOMINIQUE, Philippe (1971). * Vocabulaire boursier de la hausse et de la baisse ». La linguistique, 7, 1. (cité par Cohen 1986).

FONTENELLE, Thierry (1994). " Towards the construction of a collocational database for translation students ». Meta, 39, 1, pp. 48-56. 
GRE (1989) = Grand Robert électronique. Le Robert électronique [Ensemble d'outils d'aide à la rédaction de textes français sur disque optique compact élaboré à partir du Grand Robert de la langue française de Paul Robert dans sa nouvelle édition dirigée par Alain Rey]. Paris : Les dictionnaires Le Robert, 1989.

HAUSMANN, Franz Josef (1989). * Le dictionnaire de collocations *. Encyclopédie internationale de lexicographie, Vol 1. Berlin, W. de Gruyter. pp. 1010-1019.

HOWARTH, Peter (1993). " A Phraseological approach to academic writing *. MS. University of Leeds. (cité par Fontenelle 1994).

LAINÉ, Claude (1993). Vocabulaire combinatoire de la CFAO mécanique. Ottawa, Secrétariat d'État du Canada / Réseau international de néologie et de terminologie. (Bulletin de terminologie; 219).

LARIVIÈRE, Louise (1998). * La constitution d'un répertoire de collocations verbales : une hiérarchisation à trois niveaux n. Traduction et langues de spécialité : approches théoriques et considérations pédagogiques, sous la direction de Zélie Guével et Egan Valentine. Québec, CIRAL. (Publication B; 214). pp. 179-208.

L'HOMME, Marie-Claude (1993). * Le verbe en terminologie : du concept au contexte ". L'Actualité terminologique, 26, 2, pp. 17-19.

L'HOMME, Marie-Claude (1995). * Définition d'une méthode de recensement et de codage des verbes en langue technique : applications en traduction *. TTR : Technolectes et dictionnaires, sous la direction de Jean-Claude Boulanger, 8, 2, pp. 67-87.

L'HOMME, Marie-Claude (1997). " Méthode d'accès informatisé aux combinaisons lexicales en langue technique ". Meta, 42, 1, pp. 15-23.

MEL'CUK, Igor et al. (1984). Dictionnaire explicatif et combinatoire du français contemporain : recherches lexico-sémantiques $I$. Montréal, Les Presses de l'Université de Montréal. 
MEL'CUK, Igor, CLAS, André et Alain POLGUÈRE (1995). Introduction à la lexicologie explicative et combinatoire. Louvain-laNeuve, Éditions Duculot. (Universités francophones).

MEYER, Ingrid et Kristen MACINTOSH (1996). " Refining the terminographer's concept-analysis methods : how can phraseology help? Terminology, 3, 1, pp. 1-16.

MEYNARD, Isabelle (1998). * Combinaisons lexicales en langue de spécialité : problèmes et perspectives ". Traduction et langues de spécialité : approches théoriques et considérations pédagogiques, sous la direction de Zélie Guével et Egan Valentine. Québec, CIRAL. (Publication B; 214). pp. 123-138.

NPR $(1995)=$ Nouveau petit Robert. Le nouveau petit Robert : dictionnaire alphabétique et analogique de la langue française. Nouvelle édition du Petit Robert de Paul Robert. Texte remanié et amplifié sous la direction de Josette Rey-Debove et Alain Rey. Paris, Dictionnaires le Robert, c1993.

PAVEL, Silvia (1993). * La phraséologie en langue de spécialité. Méthodologie de consignation dans les vocabulaires terminologiques". Terminologies nouvelles, 10 , pp. 67-82.

PESANT, Ghyslaine et Estelle THIBAULT (1993). * Terminologie et cooccurrence en langue de droit ». Terminologies nouvelles, 10, pp. 23-35.

RÉSUMÉ : Valeur sémantique du verbe dans les collocations verbales spécialisées - Le présent article se propose de spécifier la valeur sémantique du verbe qui fait partie des collocations verbales spécialisées (CVS) et de caractériser, de ce fait, la nature même de cette collocation afin d'en faciliter l'identification lors de la collecte des données et, par la suite, la consignation dans un répertoire. Pour ce faire, nous établissons d'abord la place qu'occupe la collocation ou syntagme semi-figé parmi les combinaisons lexicales; puis, nous précisons la nature de la collocation, tant en langue générale qu'en langue de spécialité, en faisant ressortir la diversité de ses acceptions et de ses appellations; et, enfin, nous décrivons les caractéristiques propres et communes des trois types de verbe qui entrent dans la composition des CVS : le verbe à valeur 
métaphorique, le verbe à sens plein et le verbe à sens vide. Le statut du verbe, ainsi établi, permet de déterminer la composition des collocations verbales, en particulier en français commercial.

\begin{abstract}
The Semantic Value of the Verb in Specialized Verbal Collocations - This paper intends to specify the semantic value attached to the verb within specialized verbal collocations (SVC), and, by the same way, to characterize this type of collocation in order to facilitate its identification when collecting data, and its encoding in dictionaries. First, we will assign the place the collocation (or semi-fixed wordgroup) occupies within different sets of words; second, we will establish the nature of the collocation associated with general language as well as with specialized language, emphasizing on the diversity of its meanings and terms; third, we will describe the proper and common features of the three types of verbs used in the structure of the SVC : the figurative verb, the verb with a full meaning and the verb with an empty meaning. The status of the verb being defined will determine the composition of the verbal collocation, especially in commercial French.
\end{abstract}

Louise Larivière : Département d'études françaises, Université Concordia, 1455, boul. de Maisonneuve O., Montréal (Québec), Canada, H3G 1M8. Courriel : lariviel@magellan.ere.umontreal.ca 